\title{
La frontière comme perte d'identité, à travers l'exemple du Skåneland, entre Danemark et Suède en 1658-1660
}

\author{
Muriel Marchal \\ Université Paris-Sorbonne / Université de Stockholm
}

La frontière territoriale est considérée comme une ligne définie, qui marque la séparation entre deux territoires et relève de juridictions différentes. Il s'agit d'un objet juridique, dont la configuration institutionnalisée correspond au lieu où l'État exerce son autorité souveraine territoriale et son contrôle effectif et coercitif. La guerre remet en cause les frontières déjà établies ainsi que leur légitimité, ce qui nous amène à nous poser différentes questions: que se passe-t-il pour une province lorsqu'elle se trouve au milieu de la rivalité de deux 
pays ennemis et en guerres, qui veulent absolument la conquérir et y établir leur souveraineté? Que se passe-t-il, dans une province, lorsqu'un gouvernement impose à ses habitants, par la force, une nouvelle souveraineté? Comment est-il possible d'accepter de devenir à la fois l'allié de son ancien ennemi et l'ennemi de son ancien royaume de rattachement? Cette situation se posa pour la province du Skåneland, qui se retrouva au milieu de la rivalité dano-suédoise entre les XVIe et XVIIIe siècles. Entre 1658 et 1660 , cette province se trouva dans une situation particulièrement ambiguë, entre les royaumes de Danemark et de Suède, qui étaient, à ce moment-là, en guerre l'un contre l'autre, sans réellement savoir auquel des deux royaumes appartenir et se sentir loyale.

\section{Contexte historique}

La province du Skåneland correspond aux régions du sud actuel de la Suède, le Blekinge, la Scanie et le Halland, qui furent, jusqu'en 1658, sous l'autorité du souverain danois. En 1523, lors de la chute officielle de l'Union de Kalmar, qui avait unifié la Suède, le Danemark et la Norvège sous l'autorité du souverain danois, ces trois régions furent naturellement rattachées au royaume du Danemark pour des raisons historiques. Les statuts de cette union prévoyaient que les trois royaumes seraient mis sur un pied d'égalité et que le souverain séjournerait quatre mois dans chacun des trois royaumes. Cependant, après la mort de la reine danoise Margrethe en 1412, les souverains danois, qui se succédèrent sur le trône de l'Union de Kalmar, décidèrent de s'installer uniquement au Danemark et de considérer la Suède et la Norvège comme des provinces inférieures au Danemark. Dès lors, apparut en Suède une forte hostilité contre 
les Danois, ce qui entraîna des révoltes paysannes suédoises et, en 1520-1523, la fin de l'Union de Kalmar (Battail, Boyer, Fournier, 1992).

Entre les XVIe et XVIIIe siècles, la Scandinavie n'était pas un ensemble unifié et pacifique. Lors de la chute de cette union scandinave en 1523, le Danemark et la Suède se séparèrent officiellement et leur rivalité interne devint une hostilité ouverte sur la scène européenne, qui aboutit à un siècle et demi de guerres dano-suédoises. Ce sentiment de rivalité était dominant dans les deux royaumes scandinaves et dirigeait leur politique militaire et diplomatique. La priorité du Danemark était de reconquérir, par tous les moyens possibles, les territoires suédois, afin de faire repasser la Suède sous l'autorité unique du souverain danois. En revanche, la Suède, qui venait à peine de recouvrer son indépendance par rapport au royaume de Danemark, voulait principalement s'affirmer sur la scène diplomatique européenne, de préférence aux dépens du Danemark, son ennemi héréditaire. C'est dans ce contexte que la province du Skåneland devint un enjeu majeur de la rivalité dano-suédoise entre 1563 et 1720 .

Cette époque fut caractérisée par des guerres de nations, qui succédèrent aux guerres de seigneurs. La guerre devint alors une affaire d'État et fut de plus en plus marquée par une dimension identitaire. Il y eut sept grandes guerres directes entre la Suède et le Danemark pendant cette période 15631720 (Sundberg, 2010). La région la plus concernée par ces guerres fut le Skåneland, qui se trouve géographiquement entre la Suède et le Danemark et que les deux royaumes scandinaves se sont longtemps disputé. Cette région symbolisait plusieurs enjeux politiques et militaires très importants pour les deux 
royaumes. Le Skåneland permettait d'abord de faire peser une menace danoise permanente sur les frontières suédoises, alors que sa conquête permettrait au souverain suédois d'assurer la sécurité géographique territoriale du sud de son royaume. Cette conquête entraînerait aussi la suppression du monopole danois sur la taxation maritime du détroit du Sund et, par conséquent, une plus grande liberté et sécurité pour le commerce maritime suédois.

La Suède avait déjà commencé à conquérir une partie du Skåneland dès 1645 lors de la conclusion de la paix de Brömsebro. Ce traité fut conclu le 13 août 1645, sous la médiation de la France, qui était représentée par Gaspart Coignet de la Thuillerie, et signé par le comte Corfitz Ulfeldt, grand chancelier du Danemark, et Axel Oxenstierna, grand chancelier de Suède. Ce traité stipulait que le Danemark, en compensation de tout ce que la Suède restituait dans les pays occupés par ses troupes, devait céder aux Suédois les provinces du Härjedalen et du Jämtland, ainsi que l'île de Gotland, avec la ville de Visby et les petites îles qui en dépendaient, l'île d'Ösel avec la ville Arnsbourg et ses dépendances. Le Danemark fut également obligé de concéder la province du Halland pour une période de trente ans, comme garantie de la paix entre les deux royaumes scandinaves. Il était clairement indiqué que le souverain et le gouvernement suédois devaient respecter les anciens privilèges et maintenir la loi danoise dans la région du Halland.

Ce même traité de paix (Laursen, 1920) énonçait expressément les conditions strictes de restitution de cette province, pour obliger la Suède à respecter les termes de cession temporaire. Pourtant, le gouvernement suédois mit en 
place très rapidement une politique de suédification de cette province, sans prendre en compte les spécificités locales, afin d'empêcher tout retour possible de la souveraineté danoise dans cette province, en la rendant la plus suédoise possible. Cela marqua la naissance du mouvement des Snapphaner au Skåneland, une forme de résistance danoise anti-suédoise en réaction à cette invasion suédoise. Mais ce mouvement connut réellement son apogée en 1658, lorsque toutes les provinces du Skåneland furent cédées définitivement au roi de Suède.

\section{La première guerre danoise de Charles X Gustave}

Les années 1657-1660 sont considérées par les historiens européens comme celle de la première grande guerre du Nord. Néanmoins, il faut nuancer ce propos, car différentes guerres, indépendantes les unes des autres, opposèrent la Suède respectivement au Danemark, à la Pologne et à la Russie, sans qu'il existe d'alliance entre ces royaumes contre la Suède à cette époque. Il y eut par conséquent quatre guerres distinctes : en 1655-1660, la guerre polonaise de Charles X Gustav; en 16561661, la guerre russe de Charles X Gustave; en 1657-1658, la première guerre danoise de Charles X Gustave; et en 16581660, la seconde guerre danoise de Charles X Gustave.

L'année 1658 fut décisive dans les relations diplomatiques entre le Danemark et la Suède. Le traité de Roskilde fut signé, mettant ainsi fin à la première guerre danoise de Charles X Gustave de Suède. Le Danemark avait déclaré la guerre au royaume de Suède le 2 septembre 1657 en profitant du fait que Charles X Gustave de Suède était déjà en guerre contre la Pologne et la Russie. Frédéric III de Danemark 
espérait profiter d'un affaiblissement des défenses suédoises pour reconquérir tout le royaume de Suède, mais ce fut une tentative vaine, pendant laquelle le roi suédois fit la démonstration de sa grande puissance militaire. Le roi danois fut obligé de signer une paix très défavorable pour son royaume et céda définitivement certaines provinces au roi suédois, la région du Trondheim, dans le Nord de la Norvège, le Bohuslän, l'île de Bornholm et l'intégralité de la province du Skåneland. Le 18 février 1658, le roi Frédéric III signa le traité de Roskilde avec la Suède, marquant ainsi le début de l'ère suédoise sur le Skåneland. La frontière nationale entre le Danemark et la Suède était clairement définie par le traité de paix de Roskilde (Engelhardt, 2007), mais la réalité militaire et politique fut différente pour les habitants du Skåneland.

Ces habitants étaient officiellement considérés comme suédois par ce traité de paix, même s'ils se sentaient encore très attachés au souverain danois. Mais ils ne savaient plus à quel souverain ils devaient être fidèles, ni pour quel royaume se battre. Ils se retrouvaient perdus entre deux royaumes, sans connaître leur réelle place au sein de ce monde scandinave, en constant changement. Le dilemme auquel ils étaient confrontés était de savoir s'ils devaient se considérer comme suédois ou danois. La propagande nationale danoise les avait incités depuis plus d'un siècle à haïr le voisin et ennemi héréditaire suédois, mais à partir de 1658, ils faisaient partie intégrante de cet ennemi. Le Danemark vivait dans la haine du Suédois, et réciproquement, les habitants se posant légitimement des questions sur l'avenir de leur province qui, intégrée au royaume ennemi, devenait par la même occasion l'ennemi de son ancien royaume de rattachement. 
Il suffit pour cela de lire les Traitez de Roschild \& de Copenhague, où il est dit expressément que ces Provinces sont cedées au Roi \& à la Couronne de Suede pour lui être incorporée à perpetuité \& irrevocablement, avec tous les Droits de Souveraineté avec lesquels les Rois de Dannemarc \& de Norvegue les avoient possedez. La même chose fut repetée en termes exprès dans le Diplome de cession. Les Habitans furent tous dégagez du Serment de fidelité qu'ils avoient prêté au Roi \& à la Couronne de Dannemarc, \& obligez de le prêter au Roi \& à la Couronne de Suede. (Laursen, 1920, p. 302)

\section{La seconde guerre danoise de Charles X Gustave}

Malgré la cession de toute la province du Skåneland, le souverain danois Frédéric III ne voulait pas laisser une province aussi importante à son ennemi héréditaire suédois et fit tout son possible pour la récupérer. Le contexte historique de l'époque l'aida dans ses projets. En effet, quelques mois après la signature du traité de Roskilde, le 23 juillet 1658, le souverain suédois Charles X Gustave déclencha une nouvelle guerre contre le Danemark. Les deux royaumes recommencèrent à se battre pendant une nouvelle guerre l'un contre l'autre, qui dura deux années, et le Skåneland se trouva directement au milieu de cette rivalité et de cette guerre de force. Un élément important dans la stratégie danoise fut l'absence de déclaration de guerre de la part du roi suédois Charles X Gustave de Suède avant de passer à l'offensive. Le roi Frédéric III de Danemark décida de s'appuyer sur cet élément précis pour déclarer la nullité du traité de Roskilde et de toutes ses résolutions. Il estimait ainsi que les provinces perdues, en particulier celle du Skåneland, étaient à nouveau sous autorité danoise, mais subissaient l'occupation ennemie, qu'il considérait comme illégitime et illégale, de la part du roi de Suède (Gustafsson, 2008). 
Cependant, à cause de cette nouvelle guerre danosuédoise, le roi Frédéric III n'avait pas réellement les moyens militaires et politiques de revendiquer ces régions, ni d'envoyer des troupes pour rétablir son autorité. Il décida alors de faire appel au patriotisme des habitants pour qu'ils se soulèvent et chassent les troupes suédoises de façon que ces régions puissent réintégrer naturellement leur véritable royaume. Le 8 novembre 1658, le roi Frédéric III envoya une lettre ouverte à tous les responsables importants de la province du Skåneland, dans laquelle il écrivait :

Puisque nous ne doutons pas de votre fidèle et bonne affection à notre égard et à votre patrie, nous voulons vous rappeler et exhorter tous que vous ne devez pas du tout laisser l'occasion passer devant vous de considérer tous les moyens, par lesquels vous, avec l'aide de Dieu, pouvez-vous délivrer de la domination suédoise. (Åberg, 1965)

Il s'agissait clairement d'une invitation, de la part de Frédéric III, à provoquer une révolte interne au sein de la population, contre les autorités suédoises, au nom du royaume de Danemark. De cette manière, en se servant de son sentiment d'attachement et de loyauté envers le royaume de Danemark, le roi danois voulait lui prouver son soutien et son autorité de souverain. Pourtant, cette lettre n'eut pas l'effet escompté par le roi sur la population scanienne.

\section{Le Skåneland, au milieu de la rivalité des deux royaumes scandinaves pendant la guerre dano-suédoise}

$\mathrm{Au}$ lieu de réussir à unifier la population autour de sa personne, le roi danois la divisa en trois camps: les partisans de la 
couronne danoise, les partisans de la couronne suédoise et ceux que nous pouvons qualifier comme étant neutres, mais qui étaient très peu nombreux. Pendant la guerre de 1658-1660, il était difficile de connaître avec certitude l'avenir de cette province entre les royaumes de Danemark et de Suède. Dans ce cas précis, à cette époque, le Skåneland connaissait à la fois des problèmes de frontières matériels et immatériels. Cela eut pour conséquence que cette province perdit son identité, se trouvant alors entre deux identités distinctes, que tout opposait et qui étaient en conflit ouvert l'une contre l'autre, mais sans que cette province puisse se trouver une véritable place. Une province se définit d'abord à partir du royaume ou de la nation à laquelle elle appartient. Le Skåneland était en phase de transition, passant soudainement de l'autorité danoise à l'autorité suédoise. Toutefois, à cause de la nouvelle guerre danosuédoise de 1658-1660, le gouvernement suédois n'avait ni le temps ni les ressources nécessaires pour réellement entamer ce processus d'intégration de cette nouvelle province dans le royaume de Suède. Le Skåneland avait été danois, mais n'était pas encore suédois. Ce fut une période pendant laquelle cette province se trouva véritablement sans identité, au milieu d'un conflit armé (Gustafsson, 2008).

La nouvelle guerre dano-suédoise de 1568-1660 donna l'espoir à une partie de la population du Skåneland de pouvoir réintégrer le royaume de Danemark. L'avenir scandinave de cette province restait très incertain, ce qui posa de nombreux problèmes aux habitants, en particulier concernant leur loyauté envers le Danemark et la Suède et vit l'émergence de l'idéologie des Snapphaner. Cette rivalité, que nous pouvons observer entre le Danemark et la Suède, entre 1563 et 1720, se reflétait particulièrement sur le plan régional dans le Skåneland. Les 
Snapphaner étaient un phénomène de résistance anti-suédois contre les Suédois, qui apparut dès 1645 , lors de la cession de la province du Halland en gage de paix entre la Suède et le Danemark pour une durée de trente ans. Ce phénomène était survenu en réponse aux différentes invasions suédoises dans le Skåneland et connut réellement son apogée en 1658-1660, lorsque toutes les provinces du Skåneland passèrent de la domination danoise à la domination suédoise. Les habitants de cette province ne savaient plus à quel souverain ils devaient être loyaux et fidèles, ni quelle position adopter en cas d'invasion de l'armée d'un camp ou de l'autre.

\section{Les Snapphaner}

Le phénomène très particulier des Snapphaner apparut uniquement dans la région du Skåneland en réaction à cette situation ambiguë entre le Danemark et la Suède. Le terme Snapphane est le seul mot suédois qui est entré dans la langue française, sous la forme chenapan. Il existe plusieurs théories concernant l'origine de ce mot. Certains prétendent qu'il vient du mot allemand schnappen, qui signifie enlèvement, alors que d'autres disent que cela aurait un rapport avec les fusils qu'ils avaient toujours avec eux, comme nous pouvons l'observer sur les représentations populaires des Snapphaner. D'autres prétendent que ce mot est directement lié à sa traduction contemporaine de mâle rapide, puisque le Snapphane était en général un homme prompt à se servir de son fusil. Une autre théorie affirme que ce mot viendrait du dialecte du Halland, signifiant bandit de grand chemin, ce qui nous semble le plus vraisemblable. Selon Skansjö Stone, professeur norvégien agrégé d'histoire à l'Université de Kristianstad, ce terme 
Snapphane était utilisé comme épithète, synonyme de bandit de grand chemin (Rosén, 1943).

Le premier Snapphane fut Svend Poulsen Gjönge, qui est resté dans la légende des Snapphaner. Jeune paysan du Halland, il s'engagea dans une compagnie de l'armée danoise lors de la guerre dano-suédoise de 1643-1645. Après la fin de cette guerre, la province du Halland devint temporairement suédoise, et il fut aussitôt incorporé de force dans l'armée suédoise. Cependant, il déserta rapidement et alla se cacher dans des grottes hallandaises parce qu'il ne pouvait pas accepter cette nouvelle autorité, après avoir été aussi dévoué et loyal envers le roi de Danemark. Pendant sa clandestinité, il garda contact avec ses anciens compagnons d'armes et ses anciens officiers supérieurs danois, et commença à mettre en place un mouvement de résistance contre les Suédois, qui se propagea dans tout le Skåneland en 1657, au début de la première guerre danoise de Charles X Gustave. Pendant les deux guerres danoises de Charles X Gustave, il forma également une compagnie scanienne, qui servit au sein de l'armée danoise, prêtant ainsi main forte aux Snapphaner (Åberg, 1981).

Pour être un Snapphane, il fallait être un homme originaire du Skåneland, se sentir toujours très loyal envers le souverain danois malgré le changement d'autorité nationale de sa province, et haïr le souverain suédois au point que la perspective de devenir suédois semblait inconcevable. C'est pour cela que les Snapphaner pouvaient être considérés comme des résistants danois anti-suédois. En réalité, il s'agissait surtout de brigands, d'agriculteurs et du reste des forces scaniennes qui avaient soutenu le côté danois lors de la première guerre danoise de 1657-1658. Les Snapphaner étaient 
considérés comme la main-d'œuvre de l'armée danoise, aussi bien par les Danois que par les Suédois.

Les Snapphaner représentaient une ouverture sur les provinces du Skåneland très importante pour le roi danois. À travers ce mouvement, le roi Frédéric III savait qu'il existait quelques résistants et alliés pour les troupes danoises directement sur place. À l'exception du mouvement des Snapphaner, le roi n'avait pas de véritable contact avec ces provinces et ne connaissait ni l'ambiance locale, ni le sentiment des habitants à l'égard du royaume de Danemark. Il se basait sur cet unique élément pour affirmer ouvertement que la population locale avait besoin du retour de la souveraineté danoise dans ces provinces et le souhaitait même. Cependant, la réalité était bien différente, et la population locale était divisée en deux camps principaux et opposés: d'un côté, les Snapphaner, favorables au retour sous la souveraineté du roi danois et en résistance active contre les Suédois; et de l'autre, le reste de la population, qui voulait vivre en paix et qui, avec la victoire suédoise se profilant déjà dès le mois de février 1659 , était prête à accepter l'idée d'intégrer le royaume de Suède. En dehors de ce mouvement, nous pouvons dire qu'il n'existait pas réellement d'opposition à la nouvelle autorité suédoise dans cette région.

Les Snapphaner profitèrent de ce même doute concernant l'avenir du Skåneland pour convaincre la population du bienfondé de leurs actions. Ils restaient persuadés de la supériorité $\mathrm{du}$ système danois et des bonnes intentions du roi danois envers eux et tous les habitants de leur province. Ils croyaient sincèrement que le meilleur avenir du Skåneland se trouvait au sein du royaume de Danemark et que le souverain suédois les 
conduirait directement à leur perte, à cause de la haine très forte qui avait toujours existé entre le Danemark et la Suède. Il existait chez eux cette peur que le gouvernement suédois puisse continuer à considérer le Skåneland comme étant encore danois et donc un ennemi, et à se contenter de piller leurs richesses, sans les faire profiter des droits et privilèges spécifiques que le traité de Roskilde leur garantissait.

Le fait que la population locale était divisée en différentes allégeances posa des problèmes face aux combats acharnés des Snapphaner pour chasser les Suédois. Les Snapphaner étaient toujours considérés par une partie de la population comme des combattants de la liberté, parce que ces combattants étaient tous issus de la population locale et qu'ils se revendiquaient comme l'expression de l'humeur locale contre leur situation aussi ambiguë. Cette partie de la population locale les soutenait plus ou moins ouvertement, parce qu'elle les considérait comme des résistants, des libérateurs, voire, dans certains cas, comme des héros. Mais une autre partie de la population les considérait seulement comme des pillards et des brigands, parce qu'ils pillaient aussi bien l'armée suédoise que, dans une moindre mesure, la population locale. Ils n'adhéraient pas tous forcément aux idées des Snapphaner et ne percevaient pas le fait de changer de souverain de la même manière, parce qu'ils étaient plus préoccupés par les dégâts directs provoqués par les différentes guerres, en particulier pour leur sécurité et celle de leurs terres, à la fois contre les troupes suédoises et danoises, et aspiraient à la paix (Johannesson, 1981). 


\section{Le traité de paix de Copenhague en 1660}

En 1660, la Suède sortit à nouveau victorieuse de la guerre contre le Danemark. Le traité de Copenhague était basé en grande partie sur celui de Roskilde. Il confirmait la cession définitive des provinces du Skåneland par le Danemark à la Suède. Le traité de Copenhague en 1660 fixait de manière définitive les frontières de la Scandinavie telles que nous les connaissons aujourd'hui. Malgré deux autres grandes guerres dano-suédoises, les frontières ne bougèrent plus et le Skåneland resta suédois. La politique de suédisation se mit réellement en place après 1660 pour remplacer finalement l'identité danoise et instaurer durablement une identité suédoise au sein de cette province :

Dans l'Article 12. de celui [le traité de paix] de Copenhague elle promet de laisser les Etats desdites Provinces, tant Seculiers qu'Ecclesiastiques, dans la jouïssance des Terres qu'ils possedoient avant la guerre à juste Titre (ce qu'il faut bien remarquer) soit de succession, d'achapt, d'échange ou d'hypotheque. Elle s'engage de plus à les maintenir dans leurs anciens Droits, Privileges \& Libertez, en tant qu'ils se trouveront conformes aux Statuts \& aux Loix fondamentales de la Couronne, à laquelle ces Provinces sont incorporées à perpétuité. (Laursen, 1920, p. 563)

En danois, le mot Snapphane n'avait pas de connotation négative et était même utilisé comme une expression positive pour caractériser les guerriers paysans. Mais dès 1677, il apparaît dans différents documents que ce terme n'était plus utilisé parce qu'il était devenu synonyme de criminel. Cela date probablement du moment où les Suédois ont commencé à mettre à mort violemment ces personnes considérées comme ennemis du peuple dès 1658. Les représailles de la part du gouvernement suédois furent terribles contre les Snapphaner: 
s'ils étaient capturés par les autorités suédoises, ils connaissaient alors un destin cruel. Ces descriptions se trouvent dans l'historiographie de l'époque, tant suédoise que danoise, ainsi que dans des chroniques de guerre. Les condamnations à mort étaient exécutées très rapidement par pendaison ou par décapitation. De nombreux cas furent répertoriés comme ayant subi de longues tortures avant d'être exécutés. Le but était d'intimider et d'avertir les autres Snapphaner de ce qui allait leur arriver s'ils continuaient à se dresser contre la nouvelle autorité suédoise (Rystad, 2005).

\section{Les deux dernières tentatives danoises pour récupérer le Skåneland}

Le phénomène des Snapphaner trouva pourtant son aboutissement lors de la guerre de Scanie, skånska kriget, qui opposa à nouveau le Danemark à la Suède pour le contrôle du Skåneland entre 1675 et 1679, comme son nom l'indique. Les Snapphaner jouèrent un rôle très important pendant cette guerre en apportant un important soutien logistique aux troupes danoises. Dans les livres d'histoire, cette guerre est aussi nommée la guerre des Snapphaner. Malgré la victoire de la Suède lors de la signature du traité de paix de Lund, en septembre 1679, les deux royaumes scandinaves avaient montré leurs limites militaires, puisqu'aucun des deux royaumes n'avait été capable d'entrer dans les terres de l'autre. Ce fut la fin des prétentions danoises sur le Skåneland. La cession devenait définitive dans tous les esprits, y compris pour le roi de Danemark. Néanmoins, le processus d'intégration de cette province dans le royaume de Suède n'était pas encore abouti et il restait encore beaucoup de travail pour que les 
populations locales se sentent enfin suédoises à part entière et, plus particulièrement, pour créer un sentiment de loyauté complet envers sa personne (Battail, Boyer, Fournier, 1992).

Le phénomène des Snapphaner perdura jusqu'en 1720, date de la dernière tentative danoise pour récupérer encore cette province. Cette dernière tentative correspond à la grande guerre du Nord, entre 1700 et 1720, qui permit d'aboutir au constat que les deux royaumes scandinaves avaient définitivement perdu leur splendeur militaire. Malgré la faiblesse militaire évidente de la Suède lors de cette guerre, le Danemark n'avait pas été capable de récupérer le Skåneland ou de prendre militairement le dessus sur la Suède. La situation fut longtemps très compliquée, mais la province du Skåneland trouva finalement une identité nationale propre, lorsque les frontières furent acceptées par les deux royaumes scandinaves en 1720, cette date marquant la fin définitive des prétentions danoises sur cette province et son intégration définitive et complète dans le royaume de Suède.

\section{Le Skåneland aujourd'hui au sein de la Suède}

Le drapeau scanien actuel (rouge et jaune) est le symbole du compromis entre le Danemark et la Suède que les habitants ont fait. Il est le mélange des drapeaux danois (rouge et blanc) et suédois (bleu et jaune). Il est la représentation de la situation historique que connut le Skåneland, c'est-à-dire un compromis qui ne reniait pas ses racines danoises, mais qui acceptait sa nouvelle patrie suédoise. Les couleurs montrent bien que le Skåneland est le mélange de la Suède et du Danemark. Aujourd'hui, nous pouvons affirmer que l'identité locale 
scanienne, qui met en avant les particularités de cette province, est plus forte que l'identité nationale suédoise et a su développer ses propres particularités pour devenir une identité à part entière au sein de la Suède. Toutefois, ces deux identités arrivent à cohabiter pacifiquement, malgré leurs différences. Un habitant du Skåneland se définira d'abord comme scanien, hallandais ou blekingois, avant de dire qu'il est suédois. Les mots de Maurice Gravier, professeur émérite d'histoire nordique à la Sorbonne, montrent bien les difficultés actuelles, qui ont été causées par ce problème de frontières en 16581660 :

Les Suédois ont beaucoup souffert de la domination danoise pendant l'Union de Kalmar. Alors qu'ils glorifiaient les rois suédois, comme Gustave Vasa, qui leur avait donné l'indépendance [par rapport au Danemark], les Scaniens considèrent, aujourd'hui encore, que Gustave Vasa n'a jamais été leur souverain. À cette époque-là, ils appartenaient au Danemark depuis plus de 1000 ans. Ils regrettent aujourd'hui qu'à l'école, on n'enseigne pas aussi l'histoire de la Scanie qui, jusqu'en 1658, se confondait avec celle du Danemark. (Gravier, 1984) 


\section{Bibliographie}

ÅBERG, Alf. (1965), När Skåne blev svenskt, Stockholm, Seelig.

—. (1975), I snapphanebygd, Stockholm, Rabén \& Sjögren.

—. (1981), Snapphanarna, Lund, Signum.

-. (1995), Kampen om Skåne under försvenskningstiden, Stockholm, Natur och kultur.

BAtTAIL, Jean-François, Régis BOYER et Vincent FOURNIER. (1992), Les Sociétés scandinaves de la Réforme à nos jours, Paris, PUF.

ENGELHARDT, Paul Erik. (2007), Skåne mellem dansk og svensk: en undersøgelse af de nationale brydninger $i$ Skåne stift $i$ årene 1658 til 1679, Copenhague, Museum Tusculanum Forlag.

FRÄNGSMYR, Tore. (2004), Svensk idéhistoria: bildning och vetenskap under tusen år, vol. 1: 1000-1809, Stockholm, Natur och kultur.

GRAVIER, Maurice. (1984), Les Scandinaves : histoire des peuples scandinaves - épanouissement de leurs civilisations des origines à la Réforme, Paris, Lidis-Brepols.

GUSTAFSSON, Harald. (2008), Skåne i Danmark: en dansk historia till 1658, Göteborg, Makadam.

JoHANNESSON, Gösta. (1981), Skåne, Halland og Blekinge, Copenhague, Politikens Forlag.

LAURSEN, L. (1920), Danmark-Norges Tractater 1560-1720, Copenhague. 
LiNDROTH, Sten. (1997), Svensk lärdomshistoria. Stormaktstiden, vol. 2, Stockholm, Norstedt

Reynaert, Claude. (1972), Le Soulèvement des chenapans de Scanie (1675-1679), Lille, Revue du Nord.

RosÉn, Jerker. (1943), Hur Skåne blev svenskt, Stockholm Hugo Geber.

RYSTAD, Göran (réd.). (2005), Kampen om Skåne, Lund, Historiska media.

SANDERS, Hanne. (2008), Efter Roskildefreden 1658: Skånelandskapen och Sverige i krig och fred, Göteborg, Makadam.

SCHNAKEnBOURG, Éric et Jean-Marie MAILLEFER. (2010), La Scandinavie à l'époque moderne (fin XVe - début XIXe siècle), Paris, Belin.

SunDBERG, Ulf. (2010), Sveriges krig, Hallstavik, Svenskt militärhistoriskt bibliotek.

WeIBULL, Curt. (1921), Om det svenska och det danska rikets uppkomst, Lund. 


\title{
Résumé
}

La rivalité entre le Danemark et la Suède entraîna sept grandes guerres scandinaves entre 1563 et 1720 . Le Skåneland fut la région la plus convoitée par ces deux royaumes et se retrouva au centre des hostilités, passant de l'autorité danoise à suédoise en 1658-1660. Ce changement de souveraineté entraîna de nombreux conflits internes au sein de la population de cette région, qui perdit son identité nationale, dans un contexte de guerres dano-suédoises. Elle se trouvait entre deux souverains en guerre l'un contre l'autre, sans arriver à trouver sa place dans ce monde scandinave en constant changement. Se développa alors un phénomène typiquement scandinave, les Snapphaner, qui prônaient une utopie du Danemark aux dépens de la Suède. Intégrer le royaume ennemi héréditaire et devenir ainsi l'ennemi de son ancien royaume d'attachement, malgré des frontières officiellement établies, mais remises en cause, fut un processus très complexe pour le Skåneland en 1658-1660.

\begin{abstract}
The rivalry between Denmark and Sweden led seven important scandinavian wars, between 1563 and 1720. The Skåneland was the most coveted region by both kingdoms, and found himselv at the center of hostilites, from the danish authority to the swedish 16581660. This change of sovereignty led to many internal conflicts within the population of this region, who lost his national identity in the context of Danish-Swedish wars. This region was between two sovereigns at war against each other, without being able to find its place in this scandinavian world in constant change. Then developed a typical scandinavian phenomenon: the Snapphaner, who advocated a utopia of Denmark at the expense of Sweden. Embed the kingdom hereditary enemy and become the enemy of his former kingdom of attachment, despite borders formally established, but challenged, was a very complex process for Skåneland in 1658-1660.
\end{abstract}

